\title{
Determinants of post-operative cognitive decline in elderly people
}

J. Cartailler ${ }^{* 1,2}$, C. Loyer ${ }^{*}$, E. Vanderlynden ${ }^{3}$, R. Nizard ${ }^{4}$, C. Rabuel ${ }^{3}$, L. Coblentz Baumann ${ }^{3,5}$, C. Hourregue ${ }^{6}$, J.

$$
\text { Dumurgier }^{6}, \text { C. Paquet }{ }^{6,+}
$$

(1) Department of Anesthesiology and Intensive Care, Lariboisière - Saint Louis Hospitals, Paris, France.

(2) Paris Diderot University, Paris, France, Inserm, UMRS-942

(3) Département de médecine générale, Université de Paris

(4) Département de chirurgie orthopédique et traumatologique, APHP, Hôpital Lariboisière-Fernand Widal, Université de Paris.

(5) Patient-Centered Outcomes Research Unit, UMR 1123, Université Paris-Diderot \& Inserm

(6) Cognitive Neurology Center, Saint-Louis Lariboisière-Fernand Widal Hospital, APHP, Université de Paris INSERU1144

\section{Highlight:}

- Drop of MoCA after surgery is not associated with the type of anesthesia

- Pre-operative treatment and well-being are associated to MoCA points loss after surgery

- Per-operative complications and ketamine administration affect post-operative MoCA 


\section{Abstract:}

Background: Surgery and anesthesia can result in temporary or permanent deterioration of the cognitive functions, for which causes remain unclear.

Objectives: In this pilot study, we analyzed the determinants of cognitive decline following a non-emergency elective prosthesis implantation surgery for hip or knee.

Design: Prospective single-center study investigating psychomotor response time and changes in MoCA scores between the day before ( $D-1)$ and 2 days after ( $D+2)$ following surgery in Lariboisière Hospital (Paris, France).

Participants: 60 patients (71.9 \pm 7.1 -year-old, 72\% women) were included.

Measurements: Collected data consisted in sociodemographic data, treatment, comorbidities and type of anesthesia (local, general or both). Furthermore, we evaluated pain and well-being before as well as after the surgery using point scales.

Results: Post-operative $(D+2)$ MoCA scores were significantly lower than pre-operative ones (D-1) with a difference of median[IQR] $=2[4] p t s(p<0.001)$, we found no significant difference between locoregional and general anesthesia. Pre-operative benzodiazepine or anticholinergic treatment were also associated to a drop in MoCA scores $(p=0.006)$. Finally, the use of ketamine during anesthesia $(p=0.043)$ and the well-being $(p=0.006)$ evaluated before intervention, were both linked to a reduced cognitive impact.

Conclusion: In this pilot study, we observed a post-operative short-term cognitive decline following a lower limb surgery. We also identified pre and perioperative independent factors linked to cognitive decline following surgery. In a next stage, a larger cohort should be used to confirm the impact of these factors on cognitive decline.

Keyword: Cognitive Decline, lower limb surgery, benzodiazepine, ketamine, well-being Abbreviations: AC: Anticholinergic, BDZ: Benzodiazepine, MoCA: Montreal Cognitive Assessment, POCD: PostOperative Cognitive Decline.

\footnotetext{
* These authors contributed equally

† Corresponding author Claire Paquet: claire.paquet@inserm.fr
} 


\section{INTRODUCTION}

Post-Operative Cognitive Decline (POCD) is a major cause of mortality and morbidity costing over $\$ 150$ billion dollars yearly in health care expenses in the United States (Braunwald, et al. 2001). POCD includes postoperative delirium, NeuroCognitive Disorder (NCD) and delayed NeuroCognitive Recovery (Mahanna-Gabrielli, et al. 2019). NCD definition from the DSM-V consists in a significant cognitive decline from a previous level of performance, diagnosed at least 30 days after the surgery and assessed by standardized neuropsychological testing. Delayed NeuroCognitive Recovery are tested using the same criteria as for NCD except that the diagnosis window must be less than 30 days after the intervention (Mahanna-Gabrielli, et al. 2019).

POCD can lead to a loss of autonomy in the elderly (>60 year-old (yo)) (Fritz, et al. 2016, Wildes, et al. 2019). However, clear negative consequences of anesthesia on POCD are still debatable (Rasmussen, et al. 1999, Dokkedal, et al. 2016). Some papers speculate that POCD in the elderly is attributable to per-operative use of benzodiazepines (BDZ) and anticholinergic (AC) drugs (Pandharipande, et al. 2006, Pratico, et al. 2005), while others suggest that POCD would result from a pre-existing cerebral fragility (Wildes, et al. 2019, Dokkedal, et al. 2016). Taking into account the ageing of the population linked to a growing demand for surgery, the identification of factors increasing the risk of POCD or more severe cognitive dysfunction is needed.

Diagnosing NCD and delayed NeuroCognitive Recovery requires preoperative cognitive status along with additional stages into the routine care. Indeed, a minimal setup depends upon establishing a baseline of cognitive functions prior the surgery, then on the administration of at least a second neuropsychometric test in the post-operative period (Tsai, Sands and Leung 2010). Tools for quick assessment of POCD are for example the Confusion Assessment Method (CAM), Mini Mental State (MMS) or the Montreal Cognitive Assessment (MoCA) (Fritz, et al. 2016, Nasreddine, et al. 2005).

In particular, the MoCA test evaluates several cognitive functions which impairment is a common mechanism shared by most of POCD, including memory, concentration and verbal abstraction among others. Identifying 
POCD risk factors (drugs, comorbidity, surgical or anesthesia setups) might help targeting patients that should be followed-up, leading to a personalized care. This prospective pilot study aims to investigate the cognitive decline following a non-emergency elective hip or knee replacement, and to identify independent risk factors associated with it. Additionally, we search for factors linked with persistent cognitive decline evaluated six weeks after the surgery.

\section{METHODS}

\section{Study population}

Between February and September 2017, patients from the orthopedic department of Lariboisière Hospital, programmed for a non-emergency hip or knee replacement and who provided consent, were included in this monocentric, prospective study based on the daily clinical practice. We did not include non-French-speaking patients and those refusing to participate to the study. Based on the routine clinical practice, all patients underwent a standardized clinical examination, including medical history and physical examination, laboratory tests were performed in all subjects including chemistry panel and complete blood count. Patients underwent either a general anesthesia (GA), a locoregional anesthesia (LRA) or LRA+ propofol (LRA+P).

\section{Cognitive assessment}

To evaluate the cognitive diminution, we administered the MoCA one day before, then two days and six weeks after the surgery. The same practitioner administered MoCA tests. Additionally, the same patient never performed twice the same version of the test.

\section{Covariates}

To identify determinants of post-operative decline, we collected during the pre-, per-, and postoperative periods the following covariates: 
- Preoperative period: Age; Tobacco; Alcohol; Diabetes; Cholesterol; Hypertension (HT); Thyroids; Feeling of defective memory; Previous Neoplasia; Previous GA; Previous LRA; Cognitive Complaints; Antihypertensive treatment; Anti-diabetic treatment (per os); AC drugs; Pre-op. Antalgic; Pre-op. NSAID; BDZ; Antidepressants; MoCA (D-1) score; MoCA (D-1) evaluation duration (min); Self-evaluation of pain (D-1) based on a point scale ranging from 0 (no pain) to 10 (extreme pain); Well-being score evaluated using a custom point scale ranging from 0 (no happiness) to 10 (extreme happiness); Instrument Activity Daily Living (iADL) based on the Lawton scale.

- Peroperative period (surgery and anesthesia): Type of anesthesia as described above; Ketamine; Sufentanyl; Corticoids; Droperidol, Ephedrine; Atropine; Tranexamic acid; Clonidine chlorhydrate; Surgery duration; Occurrence of complications (see Appendix).

- Post-operative period: Antalgic; NSAID; Nefopam chlorhydrate; Pregabalin; AC; BDZ; Peri-operative complications; MoCA $(D+2, W+6)$ score; MoCA $(D+2, W+6)$ evaluation duration (min); Well-being scale; Self-evaluation of pain $(D+2, W+6)$; Well-being $(D+2)$ score.

\section{Statistical analysis}

Numerical variables were expressed by the median and interquartile range or mean and standard deviation, categorical variables were expressed as the count and percentage. For all statistical test, we chose $\alpha=0.05$ as significance level. Patient characteristic data, duration of surgery, and MoCA scores were compared using chi2, Student's t-test or Wilcoxon test as appropriate. When t-test was used, the distribution normality was assessed with the two-tailed Shapiro-Wilk and Lilliefors tests. Sensitivity of variable of interest were computed from confusion matrices.

Comparison of MoCA (D-1) scores distribution and medians between GA, LRA, and LRA+P categories were analyzed with Kolmogorov-Smirnov and Mann-Whitney tests, both two-tailed. 
For binary analysis, we associated for each patient the dummy variable 1 for the loss of at least one MoCA point between (D-1) and (D+2), 0 otherwise. We compared this response variable to binary variable and non-binary variables. To identify factors associated to a post-operative decline, we first proceeded with a univariate analysis (Chi2 for dummy variables and Logistic Regression (LR) for non-binary variable). We computed associated factors unless there were less than 5 occurrences in the entire population. Time duration of MoCA administration were compared using a two tailed paired t-test, after log-transform and normality evaluation (Shapiro-Wilk).

To investigate the impact of $A C$ and $B D Z$ treatment, we constructed two dummies variables for 'AC' $A N D$ 'BDZ' and for 'AC' OR 'BDZ' treatments. We searched for an association between these variables and cognitive decline $(\Delta$ MoCA $\geq 1)$ between $D-1$ and $D+2$.

We used a multivariate class weight corrected logistic regression model to produce a risk model for loss of at least one MoCA point between D-1 and D+2. Variables with a $p$-value $\leq 0.05$ in the univariate analysis were introduced in the multivariate model. In the case of incomplete data, patients were excluded from the multivariate analysis. We performed statistical analysis using R-studio software.

\section{RESULTS}

Sixty patients (71.9 \pm 7.1 yr., 72\% women) above 60 year-old were prospectively included in this study (Fig. 1, Tab. 1). Among them, 27 (resp. 33) underwent a non-emergency elective prosthesis hip (resp. knee) implantation surgery. The distributions of age, time of surgery, MoCA, pain and well-being scores were not different between patient from the hip and the knee groups (Kolmogorov-Smirnov test, unsignificant difference, data not shown). Therefore, for the statistical analysis we decided to consider patients that had a hip and knee surgery as a single study group. 
Patients underwent GA, $(n=27)$ or LRA $(n=14)$ or LRA+ $P(n=13)$. There was no significant difference, neither in mean nor in variance, on the $\triangle$ MoCA between D-1 and D+2 between the 3 groups of anesthesia (Fig. 2). We investigated if MoCA scores obtained one day before (D-1), two days after ( $D+2)$ and six weeks $(W+6)$ following the surgery were significantly different. MoCA score drop was 2[4] points (median[IQR], $p<0.001$ ) between (D1) and (D+2) (Fig. 3A) including 8, 14 and 19 patients who lost 1, 2 or 3 or more points respectively. We found no significant difference between D-1 and W+6 (MoCA median[IQR]: 24[3.5] versus 24 [4], $p$-value= 0.831), although among the 31 patients evaluated at 6 weeks, 32\% did not recovered their baseline level score. We observed a significant decrease in the evaluation duration of the MOCA between (D-1) and (D+2) $(p<0.001)$ and between $(D+2)$ and $(W+6)(p<0.001)$. The surgery duration did not affect the cognitive functions (median[IQR] = 70[40] min, ranging: 30 to $200 \mathrm{~min})$.

In the univariate analysis, we found a positive significant relationship between $\triangle M o C A \geq 1$ and BDZ treatment ( $p$-value $=0.047$, sensitivity $=93.8 \%), A C$ drugs ( $p$-value $=0.023$, sensitivity $=100 \%)$, 'BDZ or AC' $(p$-value $=0.006$, sensitivity $=93.8 \%)$, perioperative complications $(p$-value $=0.035$, sensitivity $=100 \%)$ and a negative significant result with the use of ketamine agent $(p$-value $=0.043$, sensitivity $=62.5 \%)$. Results are summarized in Tab. 1 . Furthermore, among non-binary variable, only well-being ( $p$-value $=0.006$, sensitivity $=94.6 \%$ ) was significantly associated with cognitive decline. Using a multivariate analysis, we found that 'well-being', 'Ketamine', 'AC' or 'BDZ', were independent risk factors associated with cognitive decline between D-1 and D+2 (see Tab. 2A). Similarly, we found that, persistent cognitive decline was associated with BDZ $(p-v a l u e=<0.001)$ and perioperative complications ( $p$-value $=0.013$ ). Additionally, we found that post-operative confusion was also associated with a W+6 cognitive decline $(p$-value $=0.002)$. Results are shown in Tab. $2 B$.

\section{DISCUSSION}


In this study, MoCA scores were significantly impacted by the surgery, with a loss of 2.25 points in average. We described that POCD were independently associated with pre-operative well-being, per-operative use of ketamine, peri-operative complications and pre-existing treatment based on either BDZ or AC drugs. In particular, well-being and ketamine had a protective effect, while complications and BDZ/AC treatments were linked to cognitive decay. Here, the type of anesthesia was not linked to cognitive decline and, persistent cognitive decline was linked to pre-existing BDZ medication and per-operative complications. Finally, MoCA evaluation duration was unchanged according the kind of anesthesia and the occurrence of POCD, however, the duration at $W+6$ was significantly shorter than $D-1$ and $D+2$ reflecting the training effect (Fig. $3 B$ ). These results suggest insight to improve perioperative cognitive protection and in particular to identify upstream of the intervention patients that could be affected by surgery or anesthesia.

Ketamine is a non-barbiturate anesthetic inhibiting acetylcholine that could lead to an increased number of delirium (Sleigh, et al. 2014). Per-operative use of ketamine is still debated, with on one side studies defending its neuroprotective and anti-inflammatory actions (Dale, et al. 2012), and on the other side studies arguing for no beneficial effects (Avidan, et al. 2017). Interestingly ketamine was shown to speed up recovery to consciousness after GA, yet possible impact on POCD was unclear (Hambrecht-Wiedbusch, Li and Mashour 2017). In the present study, per-operative ketamine administration was associated to fewer POCD, this support previous findings suggesting that administration of ketamine during a surgery reduces occurrences of POCD.

Chaiwat et al 2019 (Chaiwat, et al. 2019) reported more POCD among patients sedated with propofol, which was also suspected to decrease mean arterial pressure with a risk of brain hypoxia leading to cognitive decline (Wild, et al. 2008). However, ketamine could preserve the hemodynamic stability during propofol-based anesthesia. In our study, we compared three groups with and without propofol. We did not find any impact of the kind of anesthesia on the cognitive decline suggesting that propofol did not impact cognition in this cohort. 
Furthermore, patients who received ketamine also received propofol supporting the hypothesis of a benefic effect of the ketamine during propofol infusion. However, even if several studies are in favor of ketamine protective effect, further prospective randomized controlled studies would be needed to confirm this hypothesis.

BDZ and AC drugs have long been suspected to be involved in drug-induced cognitive decline (Starr and Whalley 1994, Bartus, et al. 1982). On one hand, BDZ act as positive allosteric modulators of GABA-A ( $\gamma$-aminobutyric acid) receptors, which results in an overall increased cortical inhibition, the latter possibly responsible for mental deterioration (Griffin III, et al. 2013). On the other hand, delirium pathway arises from a dopamine excess and acetylcholine decrease, that could be due or accentuate directly by AC drugs (Maldonado 2013). Furthermore, consequences of BDZ or AC treatment on cognition have been extensively described for speed-up age-related cognitive decay and Alzheimer's disease, not for POCD (Gage, et al. 2014, Grande, et al. 2018). In the present study, using a homogenous prospective non-emergent population, we observed detrimental effects of BDZ and AC drugs on cognitive functions. This finding supports previous results and hypothesis, however larger prospective and multicentric studies will be needed to confirm these results, allowing recommendations regarding the use of $\mathrm{BDZ}$ and $\mathrm{AC}$ drugs in non-emergent surgery. Clarifying a possible mechanisms underpinning BDZ/AC deleterious effects on sedated patients will benefit the prevention of temporary delayed NeuroCognitive Recovery as well as permanent POCD (NCD). We already know that the GABA-A agonist role of propofol is potentiated by BDZ, that could in turn promote POCD (Orser and Miller 2001). However, among our 54 patients, 15 had no propofol, yet for this subpopulation the cognitive decline was statistically undistinguishable from the 39 other patients. A regular intake of BDZ was also associated to a reduced cognitive reserve (Gage, et al. 2014), the latter linked to more POCD (Feinkohl, Winterer and Pischon 2017). Nevertheless, we found no correlation between MoCA (D-1) scores or sociocultural levels, and intake of BDZ/AC drugs. 
Per-operative complications were associated with $\triangle$ MoCA both two days and six weeks after the surgery. These findings are consistent with previous works (Greene, et al. 2009), and even more with recent works about complications following total hip arthroplasties (Aziz, et al. 2018). This suggests that an extended period under anesthesia does not affect post-operative cognition while a physiological response to a surgical complication does. Surgery-related inflammatory response has been associated to POCD (Alam, et al. 2018). Although we did not collect inflammatory-related biomarkers, we can note that among patient with peri-operative complications, only two had NSAIDs and three had ketamine.

Finally, depressed mood, in particular among the elderly, was associated with cognitive impairment (Brommelhoff, et al. 2009). Several studies outlined the link between POCD and elderly mood deterioration, assessed using Geriatric Depression Scale for patients receiving a cardiac as well as a non-cardiac surgery (Rudolph and Marcantonio 2011, Greene, et al. 2009). In our study, we found no link between cognitive decline and patient treated for depression, which agrees with these studies. Interestingly, we found that well being measured before and after the surgery were not linked to pain scores, in fact pre-operative well-being was here an independent factor associated with POCD, which was not the case for pain. These results might be explained by the fact that pain was carefully managed after the surgery (av. score $\approx 4$ ), which does mitigate POCD (Inouye, et al. 2015). In the end, it emerges from this study that well-being measured before the surgery is a better indicator of cognitive decline than post-operative pain, which suggests that the patient's state before surgery affects post-operative cognitive trajectory.

\section{Limitations and perspectives}

This study has some limitations. The patients included were very homogeneous but the inclusion criteria, the prospective methodology during a limited period led to a small number of patients. Larger multicentric studies 
would be needed to confirm or not these results and to propose recommendation regarding non-emergency arthroplasty.

In some group the number of patients was too low to provide useful data and/or multivariate analysis. However, the prospective methodology and our statistical approach allowed us to underline interesting insights about POCD. This article is the first result of a pilot study suggesting that cognitive decline occurring after a surgery could be anticipated based on pre-operative factors, yet only partially. We are performing a larger study in order to identify patients who are at risk of both delayed neurocognitive recovery and permanent cognitive decline.

\section{CONCLUSION}

To conclude, we reported a significant cognitive decline two days following a non-emergency lower limb surgery. $\mathrm{BDZ}$ or AC drugs were associated with a poor cognitive trajectory, both in short-term, but also in long-term in the case of BDZ drugs. At the same time, use of per-operative ketamine appeared to have a cognitive protective effect. Pre-operative well-being and perioperative complications were the two non-drug factors associated with positive and negative outcome respectively. Findings in the present study suggest that patient's medical prescriptions and the surgery/anesthesia smooth progress can both independently affect POCD. Nevertheless, further research, based on a larger cohort, should investigate separately factors discussed above, in particular drugs for which neurophysiological mechanisms remain unclear and the role in cognitive decline/protection is still a divisive topic.

\section{StATEMENT OF INFORMED CONSENT}

We obtained an authorization by the French data privacy administrative body 'Commission Nationale de I'Informatique et des Libertés' (CNIL), under the reference number z2c2680420w. In agreement with the ethics 
committee for this non-interventional study an oral agreement was obtained from each patient during their first medical visit.

\section{FUNDING}

Assistance Publique - Hôpitaux de Paris (APHP), Hôpital Lariboisière-Fernand Widal, Université de Paris.

\section{CONFLICTS OF INTEREST}

Pr. C. PAQUET is member of the International and National Advisory Boards of Lilly, ROCHE, Biogen. She is consultant of Fujiribio, ALZOHIS, NEUROIMMUNE and GILEAD and is involved as investigator in several clinical trials for Roche, Esai, Lilly, Biogen, Astra-Zeneca, Lundbeck, Neuroimmune.

Dr. J. DUMURGIER is investigator in several passive anti-amyloid immunotherapies and other clinical trials for Roche, Eisai, Lilly, Biogen, Astra-Zeneca, Lundbeck. Similarly, Dr. C. HOURREGUE is investigator for Roche, Eisai and Biogen.

Pr. R. NIZARD, Dr. C. RABUEL, Dr. L. COBLENTZ BAUMANN, Dr. C. LOYER, Dr. J. CARTAILLER and Dr. E. VANDERLYNDEN declare that they have no conflict of interest.

\section{AUTHORS' CONTRIBUTIONS}

Claire Paquet designed the study and analyzed the data and prepared the manuscript. Remy Nizard, Chirstophe Rabuel and Camille Loyer included the patients. Jerome Cartailler, Julien Dumurgier and Claire Paquet have performed statistical analysis and results interpretation. All authors read and validated the manuscript

\section{ACKNOWLEDGEMENTS}

Not applicable. 


\section{REFERENCES}

Alam, Azeem, Zac Hana, Zhaosheng Jin, Ka Chun Suen, and Daqing Ma. 2018. "Surgery, neuroinflammation and cognitive impairment." EBioMedicine (Elsevier).

Avidan, Michael S., Hannah R. Maybrier, Arbi Ben Abdallah, Eric Jacobsohn, Phillip E. Vlisides, Kane O. Pryor, Robert A. Veselis, et al. 2017. "Intraoperative ketamine for prevention of postoperative delirium or pain after major surgery in older adults: an international, multicentre, double-blind, randomised clinical trial." The Lancet (Elsevier) 390: 267-275.

Aziz, Keith T., Matthew J. Best, Zan Naseer, Richard L. Skolasky, Karthik E. Ponnusamy, Robert S. Sterling, and Harpal S. Khanuja. 2018. "The Association of Delirium with Perioperative Complications in Primary Elective Total Hip Arthroplasty." Clinics in orthopedic surgery 10: 286-291.

Bartus, Raymond T., R. L. 3rd Dean, Bernard Beer, and Arnold S. Lippa. 1982. "The cholinergic hypothesis of geriatric memory dysfunction." Science (American Association for the Advancement of Science) 217: 408-414.

Braunwald, Eugene, Anthony S. Fauci, Dennis L. Kasper, Stephen L. Hauser, Dan L. Longo, and J. Larry Jameson. 2001. Harrison's principles of internal medicine. McGraw Hill.

Brommelhoff, Jessica A., Margaret Gatz, Boo Johansson, John J. McArdle, Laura Fratiglioni, and Nancy L. Pedersen. 2009. "Depression as a risk factor or prodromal feature for dementia? Findings in a population-based sample of Swedish twins." Psychology and aging (American Psychological Association) 24: 373.

Chaiwat, Onuma, Mellada Chanidnuan, Worapat Pancharoen, Kittiya Vijitmala, Praniti Danpornprasert, Puriwat Toadithep, and Chayanan Thanakiattiwibun. 2019. "Postoperative delirium in critically ill surgical patients: incidence, risk factors, and predictive scores." BMC anesthesiology (BioMed Central) 19: 39.

Dale, Ola, Andrew A. Somogyi, Yibai Li, Thomas Sullivan, and Yehuda Shavit. 2012. "Does intraoperative ketamine attenuate inflammatory reactivity following surgery? A systematic review and meta-analysis." Anesthesia \& Analgesia (LWW) 115: 934-943.

Dokkedal, Unni, Tom G. Hansen, Lars S. Rasmussen, Jonas Mengel-From, and Kaare Christensen. 2016. "Cognitive functioning after surgery in middle-aged and elderly Danish twins." Anesthesiology: The Journal of the American Society of Anesthesiologists (The American Society of Anesthesiologists) 124: 312-321.

Feinkohl, I., G. Winterer, and T. Pischon. 2017. "Hypertension and risk of post-operative cognitive dysfunction (POCD): A systematic review and meta-analysis." Clinical practice and epidemiology in mental health: CP \& EMH (Bentham Science Publishers) 13: 27.

Fritz, Bradley A., Philip L. Kalarickal, Hannah R. Maybrier, Maxwell R. Muench, Doug Dearth, Yulong Chen, Krisztina E. Escallier, Arbi Ben Abdallah, Nan Lin, and Michael S. Avidan. 2016. "Intraoperative electroencephalogram suppression predicts postoperative delirium." Anesthesia and analgesia (NIH Public Access) 122: 234.

Gage, Sophie Billioti, Yola Moride, Thierry Ducruet, Tobias Kurth, Hélène Verdoux, Marie Tournier, Antoine Pariente, and Bernard Bégaud. 2014. "Benzodiazepine use and risk of Alzheimer's disease: case-control study." Bmj (British Medical Journal Publishing Group) 349: g5205.

Grande, G., I. Tramacere, D. L. Vetrano, S. Pomati, C. Mariani, and G. Filippini. 2018. "Use of benzodiazepines and cognitive performance in primary care patients with first cognitive complaints." International psychogeriatrics (Cambridge University Press) 30: 597-601. 
Greene, Nathaniel H., Deborah K. Attix, B. Craig Weldon, Patrick J. Smith, David L. McDonagh, and Terri G. Monk. 2009. "Measures of executive function and depression identify patients at risk for postoperative delirium." Anesthesiology: The Journal of the American Society of Anesthesiologists (The American Society of Anesthesiologists) 110: 788-795.

Griffin III, Charles E., Adam M. Kaye, Franklin Rivera Bueno, and Alan D. Kaye. 2013. "Benzodiazepine pharmacology and central nervous system--mediated effects." The Ochsner Journal (the Academic Division of Ochsner Clinic Foundation) 13: 214-223.

Hambrecht-Wiedbusch, Viviane S., Duan Li, and George A. Mashour. 2017. "Paradoxical EmergenceAdministration of Subanesthetic Ketamine during Isoflurane Anesthesia Induces Burst Suppression but Accelerates Recovery." Anesthesiology: The Journal of the American Society of Anesthesiologists (The American Society of Anesthesiologists) 126: 482-494.

Inouye, Sharon K., Thomas Robinson, Caroline Blaum, Jan Busby-Whitehead, Malaz Boustani, Ara Chalian, Stacie Deiner, et al. 2015. "Postoperative delirium in older adults: best practice statement from the American Geriatrics Society." Journal of the American College of Surgeons (Elsevier) 220: 136-148.

Mahanna-Gabrielli, Elizabeth, Katie J. Schenning, Lars I. Eriksson, Jeffrey N. Browndyke, Clinton B. Wright, Lis Evered, David A. Scott, et al. 2019. "State of the clinical science of perioperative brain health: report from the American Society of Anesthesiologists Brain Health Initiative Summit 2018." British Journal of Anaesthesia (Elsevier).

Maldonado, José R. 2013. "Neuropathogenesis of delirium: review of current etiologic theories and common pathways." The American Journal of Geriatric Psychiatry (Elsevier) 21: 1190-1222.

Nasreddine, Ziad S., Natalie A. Phillips, Valérie Bédirian, Simon Charbonneau, Victor Whitehead, Isabelle Collin, Jeffrey L. Cummings, and Howard Chertkow. 2005. "The Montreal Cognitive Assessment, MoCA: a brief screening tool for mild cognitive impairment." Journal of the American Geriatrics Society (Wiley Online Library) 53: 695-699.

Orser, Beverley A., and Donald R. Miller. 2001. "Propofol-benzodiazepine interactions: insights from a "bench to bedside" approach." Canadian Journal of Anesthesia/Journal canadien d'anesthésie (Springer) 48: 431-434.

Pandharipande, Pratik, Ayumi Shintani, Josh Peterson, Brenda Truman Pun, Grant R. Wilkinson, Robert S. Dittus, Gordon R. Bernard, and E. Wesley Ely. 2006. "Lorazepam is an independent risk factor for transitioning to delirium in intensive care unit patients." Anesthesiology: The Journal of the American Society of Anesthesiologists (The American Society of Anesthesiologists) 104: 21-26.

Pratico, C., D. Quattrone, T. Lucanto, A. Amato, O. Penna, C. Roscitano, and V. Fodale. 2005. "Drugs of anesthesia acting on central cholinergic system may cause post-operative cognitive dysfunction and delirium." Medical hypotheses (Elsevier) 65: 972-982.

Rasmussen, L. S., Anni Steentoft, H. Rasmussen, P. A. Kristensen, and J. T. Moller. 1999. "Benzodiazepines and postoperative cognitive dysfunction in the elderly. ISPOCD Group. International Study of Postoperative Cognitive Dysfunction." British journal of anaesthesia (Elsevier) 83: 585-589.

Rudolph, James L., and Edward R. Marcantonio. 2011. "Postoperative delirium: acute change with long-term implications." Anesthesia and analgesia (NIH Public Access) 112: 1202.

Sleigh, Jamie, Martyn Harvey, Logan Voss, and Bill Denny. 2014. "Ketamine--More mechanisms of action than just NMDA blockade." Trends in anaesthesia and critical care (Elsevier) 4: 76-81.

Starr, John M., and Lawrence J. Whalley. 1994. "Drug-induced dementia." Drug Safety (Springer) 11: 310-317. 
Tsai, Tiffany L., Laura P. Sands, and Jacqueline M. Leung. 2010. "An update on postoperative cognitive dysfunction." Advances in anesthesia (Elsevier) 28: 269-284.

Wild, Katherine, Diane Howieson, Frank Webbe, Adriana Seelye, and Jeffrey Kaye. 2008. "Status of computerized cognitive testing in aging: a systematic review." Alzheimer's \& Dementia (Elsevier) 4: 428-437.

Wildes, Troy S., Angela M. Mickle, Arbi Ben Abdallah, Hannah R. Maybrier, Jordan Oberhaus, Thaddeus P. Budelier, Alex Kronzer, et al. 2019. "Effect of electroencephalography-guided anesthetic administration on postoperative delirium among older adults undergoing major surgery: the ENGAGES randomized clinical trial." Jama (American Medical Association) 321: 473-483. 


\section{GRAPHICS}

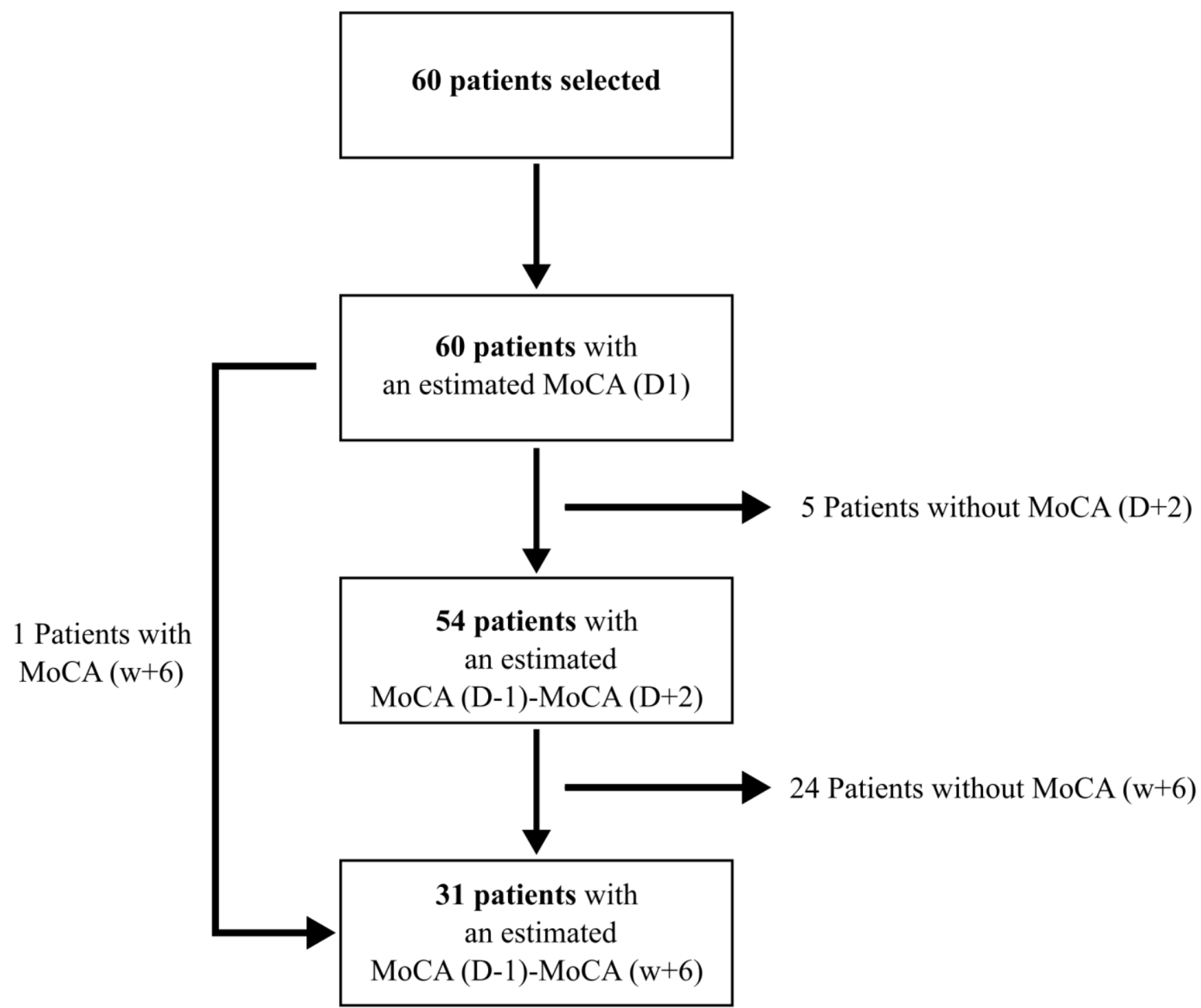

Figure 1: Flowchart 


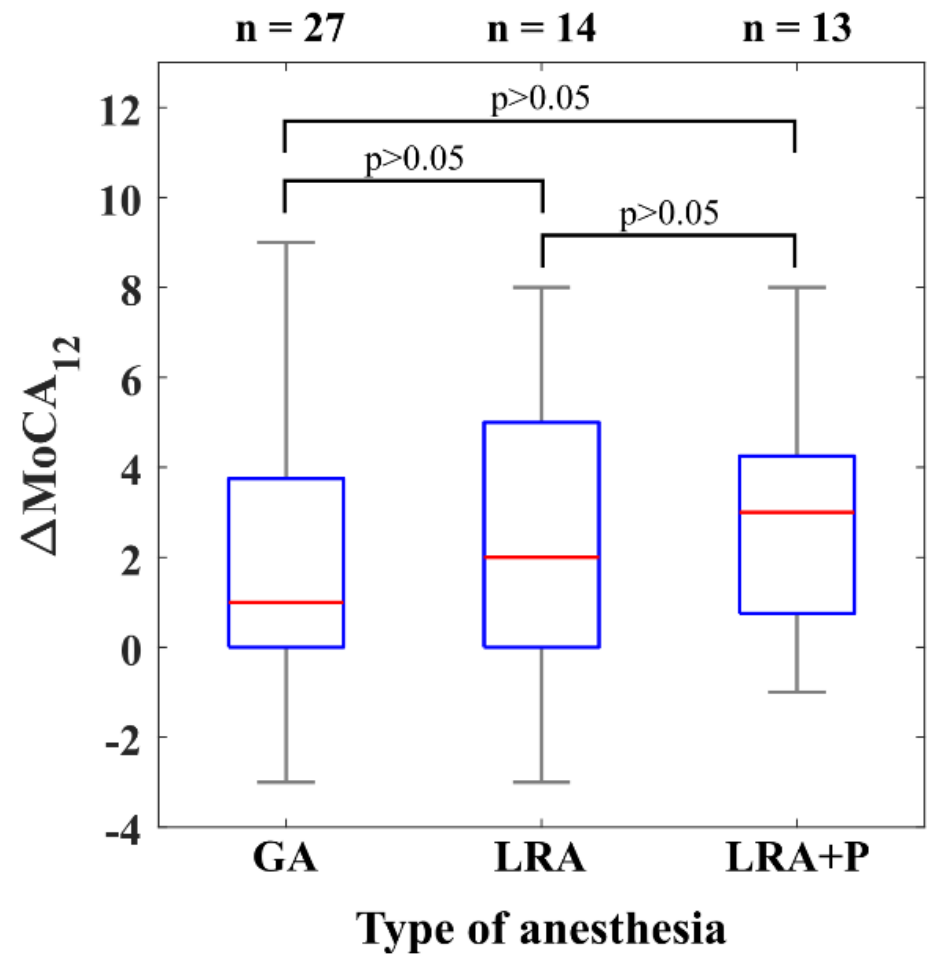

Figure 2: Distribution of $\triangle M o C A$ between $D-1$ and $D+2$ for the three types of anesthesia (GA, LRA, LRA+Propofol) showing no significant difference in (Wilcoxon test).
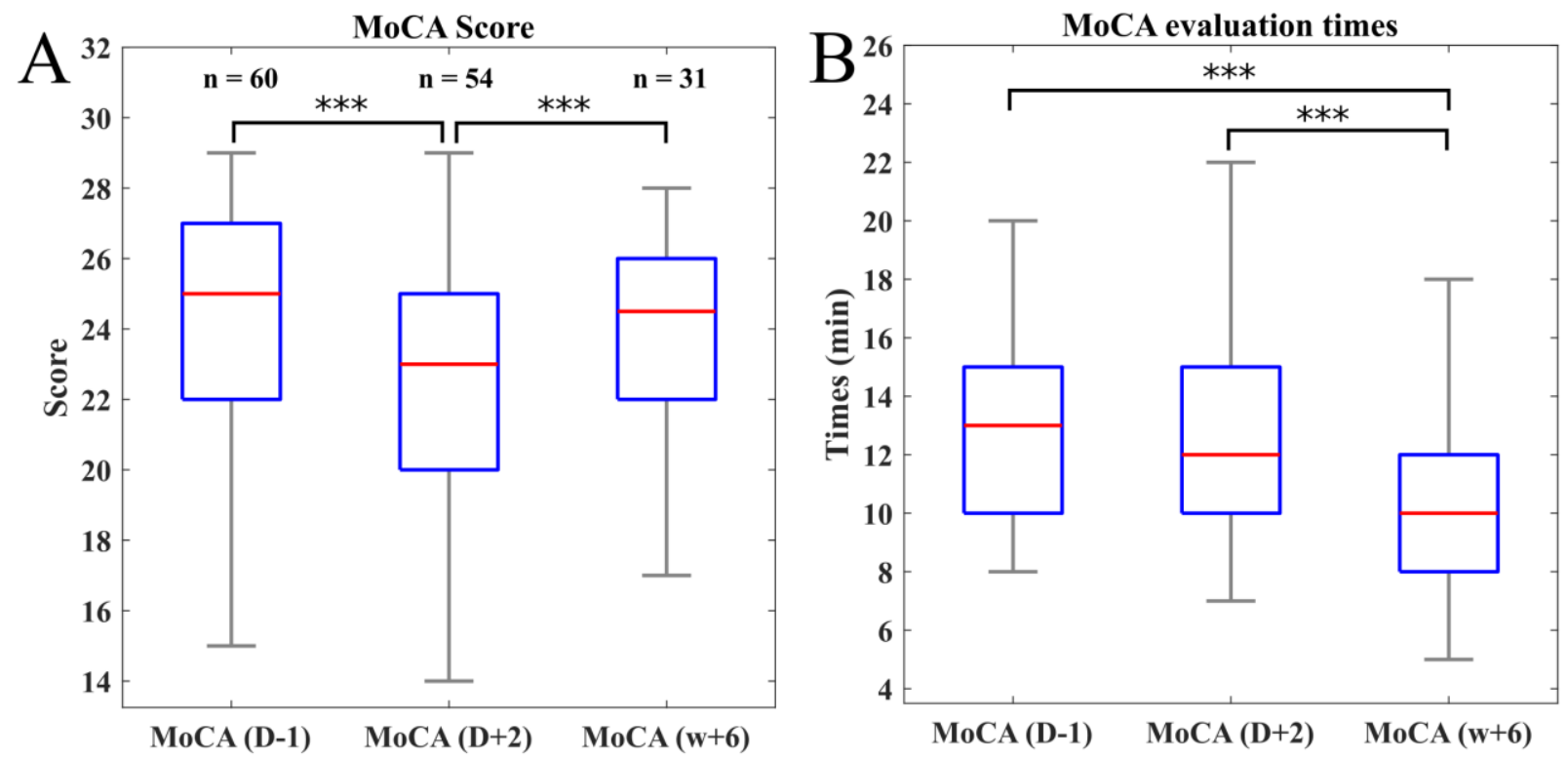
Figure 3: Distribution of MoCA score (A) and evaluation time (B) for three different periods:

one day before (D-1), two days $(D+2)$ and six weeks $(w+6)$ following the surgery and anesthesia.

Table 1: Comparison of population characteristic data between the patient with $\triangle M o C A \geq 1$ pt and patients with no point loss. Binary data are number and percentage, while non binary data are shown in median and interquartile range (IQR). NSAIDs; Nonsteroidal anti-inflammatory drugs, + includes the following complications: failed LRA - modification of the surgery - Hemorrhage - incisors defect (see Supplementary).

\begin{tabular}{|c|c|c|c|c|c|c|c|}
\hline \multirow[t]{2}{*}{ VARIABLES } & \multicolumn{2}{|c|}{ All $(n=54)$} & \multicolumn{2}{|c|}{$\begin{array}{l}\text { Unaffected MoCA } \\
\text { group }(n=16)\end{array}$} & \multicolumn{2}{|c|}{$\begin{array}{l}\text { Altered MoCA group } \\
\qquad(n=38)\end{array}$} & \multirow[b]{2}{*}{ p-value } \\
\hline & $\begin{array}{c}\mathrm{n} \text { or } \\
\text { median }\end{array}$ & $\begin{array}{l}\text { (\%) or } \\
\text { [IQR] }\end{array}$ & $\begin{array}{c}\mathrm{n} \text { or } \\
\text { median }\end{array}$ & $\begin{array}{l}\text { (\%) or } \\
\text { [IQR] }\end{array}$ & $\begin{array}{c}\mathrm{n} \text { or } \\
\text { median }\end{array}$ & $\begin{array}{l}\text { (\%) or } \\
\text { [IQR] }\end{array}$ & \\
\hline \multicolumn{8}{|l|}{ PRE-OPERATIVE } \\
\hline Age (yrs.) & 70.5 & {$[7.5]$} & 69.5 & {$[5.5]$} & 72 & {$[7.8]$} & 0.259 \\
\hline Sex (female) & 39 & $72.2 \%$ & 9 & $56.3 \%$ & 30 & $79.0 \%$ & 0.089 \\
\hline Sociocultural level & 3 & [2] & 3 & [2] & 3.5 & [2] & 0.571 \\
\hline $\begin{array}{l}\text { Instrumental Activities of Daily } \\
\text { Living }\end{array}$ & 4 & {$[0]$} & 4 & [0] & 4 & {$[0]$} & 0.731 \\
\hline Smoker & 8 & $14.8 \%$ & 2 & $12.5 \%$ & 6 & $15.8 \%$ & 0.756 \\
\hline Alcohol (0/1) & 6 & $11.1 \%$ & 3 & $18.8 \%$ & 3 & $7.9 \%$ & 0.246 \\
\hline Hypertension (0/1) & 35 & $64.8 \%$ & 12 & [75] & 23 & $60.5 \%$ & 0.309 \\
\hline Cholesterol & 24 & $44.4 \%$ & 8 & {$[50]$} & 16 & $42.1 \%$ & 0.594 \\
\hline Diabetes (0/1) & 11 & $20.4 \%$ & 3 & $18.8 \%$ & 8 & $21.1 \%$ & 0.848 \\
\hline Thyroid & 7 & $13.0 \%$ & 1 & $6.3 \%$ & 6 & $15.8 \%$ & 0.341 \\
\hline Cognitive trouble in family $(0 / 1)$ & 6 & $11.1 \%$ & 1 & $6.3 \%$ & 5 & $13.2 \%$ & 0.461 \\
\hline Nb. of drug & 4.5 & [5] & 3 & [3.8] & 5 & [4] & 0.204 \\
\hline $\begin{array}{l}\text { Anti-hypertensive treatment } \\
(0 / 1)\end{array}$ & 1 & [1] & 1 & {$[0.5]$} & 1 & [1] & 0.163 \\
\hline Anti-diabetic treatments $(0 / 1)$ & 10 & $18.5 \%$ & 3 & $18.8 \%$ & 7 & $18.4 \%$ & 0.977 \\
\hline $\begin{array}{l}\text { Preexisting NSAIDs } \\
\text { treatment }(0 / 1)\end{array}$ & 16 & $29.6 \%$ & 3 & $18.8 \%$ & 13 & $34.2 \%$ & 0.256 \\
\hline $\begin{array}{l}\text { Preexisting antalgic } \\
\text { treatment }(0 / 1)\end{array}$ & 42 & $77.8 \%$ & 11 & $68.8 \%$ & 31 & $81.6 \%$ & 0.3 \\
\hline $\begin{array}{l}\text { Preexisting antidepressant } \\
\text { treatment }(0 / 1)\end{array}$ & 7 & $13.0 \%$ & 1 & $6.3 \%$ & 6 & $15.8 \%$ & 0.341 \\
\hline $\begin{array}{l}\text { Preexisting benzodiazepine } \\
\text { treatment }(0 / 1)\end{array}$ & 13 & $24.1 \%$ & 1 & $6.3 \%$ & 12 & $31.6 \%$ & 0.047 \\
\hline $\begin{array}{l}\text { Preexisting anticholinergic } \\
\text { treatment }(0 / 1)\end{array}$ & 10 & $18.5 \%$ & 0 & $0.0 \%$ & 10 & $26.3 \%$ & 0.023 \\
\hline $\begin{array}{l}\text { Preexisting anticholinergic } O R \\
\text { benzodiazepine treatment }(0 / 1)\end{array}$ & 18 & $33.3 \%$ & 1 & $6.3 \%$ & 17 & $44.7 \%$ & 0.006 \\
\hline
\end{tabular}




\begin{tabular}{|c|c|c|c|c|c|c|c|}
\hline MoCA score (D-1) & 25 & [4.8] & 24 & {$[3.2]$} & 25.5 & [4] & 0.035 \\
\hline Time to perform MoCA (min) & 13 & [5] & 14 & [3] & 12 & [5] & 0.683 \\
\hline Memory complaint $(0 / 1)$ & 16 & $29.6 \%$ & 3 & $18.8 \%$ & 13 & $34.2 \%$ & 0.256 \\
\hline Pain scale (D-1) & 0 & [5] & 0 & {$[0.2]$} & 0 & [5] & 0.084 \\
\hline Well-being (D-1) & 8 & {$[2]$} & 8 & [1] & 7.5 & {$[2.5]$} & 0.006 \\
\hline Nb. Previous general anesthesia & 2 & [3] & 1.5 & {$[2.2]$} & 2 & [2] & 0.092 \\
\hline $\begin{array}{l}\text { Nb. Previous loco-regional } \\
\text { anesthesia }\end{array}$ & 0 & {$[0]$} & 0 & {$[1.2]$} & 0 & [0] & 0.259 \\
\hline \multicolumn{8}{|l|}{ PER-OPERATIVE } \\
\hline General anesthesia (0/1) & 27 & $50.0 \%$ & 8 & $50.0 \%$ & 19 & $50.0 \%$ & 0.999 \\
\hline Loco-regional anesthesia $(0 / 1)$ & 14 & $25.9 \%$ & 4 & $25.0 \%$ & 10 & $26.32 \%$ & 0.712 \\
\hline Loco-regional + propofol (0/1) & 13 & $24.1 \%$ & 4 & $25.0 \%$ & 9 & $23.68 \%$ & 0.690 \\
\hline Surgery duration (min) & 70 & [40] & 70 & [35] & 75 & [40] & 0.812 \\
\hline Propofol & 39 & $72.2 \%$ & 11 & $68.8 \%$ & 28 & $73.7 \%$ & 0.712 \\
\hline Sufentanyl & 30 & $55.6 \%$ & 9 & $56.3 \%$ & 21 & $55.3 \%$ & 0.947 \\
\hline Atracurium & 24 & $44.4 \%$ & 7 & $43.3 \%$ & 17 & $44.7 \%$ & 0.947 \\
\hline Ropivacaine & 25 & $46.3 \%$ & 9 & $56.3 \%$ & 16 & $42.1 \%$ & 0.341 \\
\hline Marcaine/bupivacaine & 21 & $38.9 \%$ & 5 & $31.3 \%$ & 16 & $42.1 \%$ & 0.455 \\
\hline Ketamine & 11 & $20.4 \%$ & 6 & $37.5 \%$ & 5 & $13.2 \%$ & 0.043 \\
\hline Ephedrine & 25 & $46.3 \%$ & 6 & $37.5 \%$ & 19 & $50.0 \%$ & 0.4 \\
\hline Atropine & 8 & $14.8 \%$ & 3 & $18.75 \%$ & 5 & $13.2 \%$ & 0.597 \\
\hline Clonidine chlorhydrate & 6 & $11.1 \%$ & 2 & $12.5 \%$ & 4 & $10.5 \%$ & 0.833 \\
\hline Droperidol & 30 & $55.6 \%$ & 9 & $56.3 \%$ & 21 & $55.3 \%$ & 0.947 \\
\hline Tranexamic Acid & 29 & $53.7 \%$ & 6 & $37.5 \%$ & 23 & $60.5 \%$ & 0.121 \\
\hline Per-operative complications ${ }^{\dagger}$ & 8 & $14.8 \%$ & 0 & $0.0 \%$ & 8 & $21.1 \%$ & 0.047 \\
\hline \multicolumn{8}{|l|}{ POST-OPERATIVE } \\
\hline Post-operative Confusion & 7 & $13.0 \%$ & 0 & $0.0 \%$ & 7 & $18.4 \%$ & 0.066 \\
\hline Pain scale $(D+2)$ & 4.2 & [4] & 2.8 & {$[5.2]$} & 4.8 & [4] & 0.244 \\
\hline Post-operative well being & 8 & [3] & 9 & {$[2.5]$} & 8 & [2] & 0.3 \\
\hline MoCA at $D+2$ & 23 & [5] & 24.5 & {$[4.2]$} & 22 & [5] & 0.014 \\
\hline Time to perform MoCA (min) & 12 & {$[5]$} & 11 & [4.2] & 12 & [5] & 0.294 \\
\hline
\end{tabular}

Table 2: Factors associated with cognitive decline at $D+2$ and $\mathbf{w + 6 .}$

2A Sensitivity of risk factors of cognitive decline at D+2 computed with their significance computed from multivariate logistic regression for Ketamine, Wellbeing and 'AC or BDZ'

\begin{tabular}{|l|c|c|}
\hline VARIABLE & SENSITIVITY & P-VALUE \\
\hline Per-op. complication & $100 \%$ & - \\
\hline Well-being $=0$ & $94.6 \%$ & 0.012 \\
\hline
\end{tabular}

2B Patient characteristic associated to cognitive decline at six weeks $(w+6)$ using a chi2 analysis 


\begin{tabular}{|l|l|l|}
\hline AC OR BDZ & $93.8 \%$ & $<0.001$ \\
\hline Ketamine & $62.5 \%$ & 0.026 \\
\hline
\end{tabular}

\section{Appendix:}

\begin{tabular}{|l|l|l|l|l|}
\hline \multicolumn{1}{|c|}{ Statistic/ } & \multicolumn{1}{c}{ AG } & \multicolumn{2}{c|}{ ALR } & \multicolumn{2}{c|}{ ALR + P } & \multicolumn{1}{c|}{ All } \\
\hline $\mathbf{n}$ & 27 & 14 & 13 & 54 \\
\hline Minimum & -3 & -3 & -1 & -3 \\
\hline Maximum & 9 & 8 & 8 & 9 \\
\hline Mean & 1.89 & 2.36 & 2.92 & 2.25 \\
\hline Standard deviation (n-1) & 3.18 & 3.30 & 2.57 & 3.05 \\
\hline
\end{tabular}

Table A1: MoCA D-1/D+2 drop statistics for the three types of anesthesia

\begin{tabular}{|l|l|}
\hline Type of peroperative complications encountered & $\mathbf{n}$ \\
\hline
\end{tabular}

LRA installation failed 2

A bilateral hip surgery became a hip and knee surgery

Hemorrhage 1 
Incisors broken during oro-tracheal intubation

Table A2: List of peroperative complications 